\title{
An Improved Reconstruction methods of Compressive Sensing Data Recovery in Wireless Sensor Networks
}

\author{
Sai Ji ${ }^{1,2}$, Liping Huang ${ }^{2}$, Jin Wang ${ }^{2}$, Jian Shen ${ }^{2}$ and Jeong-Uk Kim ${ }^{3}$ \\ 1 The Aeronautic Key Laboratory for Smart Materials and Structures, Nanjing \\ University of Aeronautics and Astronautics, Nanjing, China, 210016 \\ 2 Jiangsu Engineering Center of Network Monitoring, Nanjing University of \\ Information Science and Technology, Nanjing, China, 210044 \\ ${ }^{3}$ Department of Energy Grid, Sangmyung University, Seoul 110-743, Korea
}

\begin{abstract}
Energy consumption is a critical problem affecting the lifetime of wireless sensor networks (WSNs) in structural health monitoring (SHM). A huge original acquisition data was transmitted between nodes which occupy a large amount of communication bandwidth, and even lead to paralysis of WSNs. Thus, data compression to reduce network traffic and energy loss before transmission is necessary. A number of traditional techniques have proposed to solve this issue by sampling the full signal and then taking compression process. But it spends a lot of processing time. In this paper, we establish suitability compressive sensing (CS) to address some challenges using WSN. Through the improvement of reconstruction algorithm and the experimental demonstration, the application of this method could ensure the accuracy of the data as well as balance the network energy consumption. Moreover, it can also reduce the cost of data storage and transmission which makes a certain contribution to the quality for SHM.
\end{abstract}

Keywords: compressive sensing; wireless sensor networks; structural health monitoring; reconstruction algorithm

\section{Introduction}

With the development of WSN applications in structural health monitoring, data compression technology for SHM has been attracted a growing number of scholars' attention. However, formerly, we arranged a large number of sensor nodes using a high frequency sampling rate for data acquisition and real-time monitoring, while as we all know that wireless sensor nodes have limited energy. Thus, it is necessary to get efficient compression in order to balance the network energy and reduce data storage or transmission cost.

SHM researchers proposed some compression algorithm [1-3] to achieve high compression efficiency. However, all methods above belong to the traditional framework for data compression which is firstly to sample the full signal and then compress it. Recently, a new data compression method named compressive sensing (CS)[4,5] which acquires data in compressed form directly by using special sensors has been presented initially used for image process. With the development of CS theory, it has been applied to WSNs, medical imaging, remote sensing [6], digital camera and so on. In the SHM, a few researchers [7, 8] applied it to the reconstruction of structural modal shape and analyzed plate structures.

A lot of studies have shown that CS theory is applicable for the structural monitoring narrowband signal compression which could enhance the robustness of the network data 
transmission, and then it has a good prospect of application in the field of WSNs for SHM. Currently, though some CS applications have been existed in the SHM, it is still at the initial stage.

In this article, we mainly focused on the reconstruction methods research. Comparing with the existing methods, we proposed an improved reconstruction data algorithm of OMP in the CS application framework which is suitable for the SHM based on the WSN nodes. Through the experimental demonstration, it is able to ensure the data accuracy, balance the network energy consumption, and reduce the cost of data storage and transmission.

The rest of the paper is organized as follows. Section 2 describes the compressive sensing theory which is the basement of the CS application. The improved OMP that is Threshold Orthogonal Matching Pursuit (TOMP) algorithm is also proposed in Section 2. Section 3 presents the simulation results and analysis. And Section 4 concludes the paper.

\section{Compressive Sensing Theory}

Mainly, CS theory includes three parts: the sparse representation of the signal, the measurement matrix ensuring the data minimal information loss which should be satisfied the Restricted Isometry Property (RIP) and the reconstruction algorithm using the no-distortion observed value to reconstruct signals. Among them, reconstruction algorithm is an important factor in CS.

\subsection{Signal Sparsity and the Sparse Representation}

Signal sparsity is an important prerequisite for CS theoretical foundation. The essence of CS [9] is using an irrelevant measurement $(M \times N, M<N)$ with a matrix transform base (dimension) to put the original high-dimensional sparse signal or approximate sparse signal sequence $N \times 1$ project in a low dimensional space ( $M$ dimension) to achieve signal compression.

Suppose that a measurement matrix $\Phi \in R^{M \times N}(M<<N)$ is introduced to produce compressed sensing coefficients $\mathrm{y}$, then for a sparse signal $x \in R^{N}$, the linear measuring values in the measurement matrix $y \in R^{M}$ could be defined as (1), where $\Phi$ is called a measurement matrix.

$$
y=\Phi x
$$

However, we all know that nature signal is usually not absolutely sparse, but if it can be as approximate spare signal in some transform domains such as Fourier domain, Wavelet domain and QuBo domain, we considered it is compressible signal. So through one of the orthogonal transformations $\Psi$, we can achieve sparse representation (2).

$$
y=\Phi x=\Phi \Psi \alpha=\Theta \alpha
$$

Where $\Theta=\Phi \Psi$ is a $M \times N$ sensing matrix, $\Phi$ represents a $M \times N$ measurement matrix and $\Psi$ is a $N \times N$ transformation matrix.

Formula(2) can be regarded as the linear projection of original signal $x$ with $\Phi$, and it could be also viewed as the linear projection of transform decomposition coefficients $\alpha$ in $\Theta$. If $\mathrm{y}$ and $\Theta=\Phi \Psi$ meet with the RIP [10], K-sparse decomposition coefficients $\alpha$ can be reconstructed by solving the $l_{0}$ norm [11] from y as (3).

$$
\hat{\alpha}=\arg \min \|\alpha\|_{0} \quad \text { s.t. } \quad \Theta \alpha=y
$$


where $\hat{\alpha}$ is the only exact solution of decomposition coefficients $\alpha$. Finally, exact solution $\hat{x}$ can be obtained by reconstructing $\hat{\alpha}$ under the orthogonal transform basis $\Psi$ shown as (4):

$$
\hat{x}=\Psi \hat{\alpha}
$$

\subsection{Measurement Matrix Selections in CS}

In the exact solution procedure to reconstruct original signal, sensing matrix $\Theta=\Phi \Psi$ must meet the RIP which is proposed by Candes and Tao [10], and its definition is as follows:

Definition, Introducing any $\mathrm{K}$ sparse signal $x$ and constant $\delta_{K} \in(0,1)$, sensing matrix $\Theta$ should be fulfill with the equation (5):

$$
1-\delta_{K} \leq \frac{\|\Theta x\|_{2}^{2}}{\|x\|_{2}^{2}} \leq 1+\delta_{K}
$$

To further demonstrate the intrinsic relationship between $\Psi$ and $\Phi$ while $\Theta=\Phi \Psi$ met with the RIP, Baraniuk [12] proposed that the equivalence condition of the RIP which is $\Phi$ irrelevant with $\Psi$, i.e., the $\Theta$ row vector cannot be represented by $\Psi$ column vector, and the $\Psi$ row vector cannot be represented by $\Theta$ column vector.

Therefore, we select tectonic $\Phi$ measurement matrix in the orthogonal base matrix $\Psi$ which is fixed to make $\Theta=\Phi \Psi$ satisfy with RIP in this article.

\subsection{Reconstruction Algorithm Selection in CS}

Reconstruction algorithms are CS theory's core which using the value of the measurement vector $\mathrm{y}$ in $\mathrm{M}$ dimension to reconstruct the sparse signal in the length $\mathrm{N}$. Candes proved that the signal reconstruction problem can be solved by solving the minimum $l_{0}$ norm which is shown on the formula (3), so that all signal reconstructions which solved by the $l_{0}$ norm can be resolved. Currently, the algorithm is mainly divided into three categories which are greedy algorithm [13,14], convex optimization algorithms[15] and the sparse Bayesian statistical optimization algorithm[16].

Greedy Pursuit algorithm is selected a local optimal solution by each iteration to gradually approximate with original signals which made the reconstruction realization simply and fast. So it is suitable for the lower dimension small-scale signal problem. The most typical algorithms are Matching Pursuit (MP) algorithm [13] and Orthogonal Matching Pursuit (OMP) algorithm [14]. In this paper, we improved the OMP algorithm and used it as the stabled reconstruction method.

\subsection{Improved OMP Algorithm (TOMP)}

We improved the OMP algorithm and used it as the stabled reconstruction method which had a faster convergence. Table 1 shows the improved algorithm which called the TOMP (Threshold Orthogonal Matching Pursuit).

Table 1. TOMP Algorithm

\begin{tabular}{l} 
Algorithm 1 TOMP algorithm for the signal reconstruction \\
\hline Input: residuals $\mathrm{r} 0=\mathrm{v}$, Index set $\Lambda 0=\varnothing$, Iteration count $\mathrm{t}=1$; \\
Output: the elements in $\Lambda \mathrm{m}$ which are the nonzero value indicators of the \\
recovery signal $\mathrm{x}^{*}$, and noticing that the $\lambda \mathrm{j}-$ th element value in the $\mathrm{s}^{*}$ is equal to
\end{tabular}


the $\mathrm{j}$-th element value in $\mathrm{xt}$.

Step 1: Find indicator $\lambda t$, to meet the following optimization problem: $\lambda t=a r g$ $\max \mathrm{j}=1, \ldots, \mathrm{d}|<\mathrm{rt}-1, \varphi \mathrm{j}>|$;

Step 2: Expansion of the index set $\Lambda \mathrm{t}=\Lambda \mathrm{t}-1 \cup\{\lambda \mathrm{t}\}$ and the matrix $\Phi \mathrm{t}=[\Phi \mathrm{t}-1 \varphi \mathrm{t} \mathrm{t}]$, $\Phi 0$ is a empty matrix;

Step 3: Solving the Least squares problem: $x t=\arg \min x\|v-\Phi t x\| 2$;

Step 4: Calculate the new signal estimation and residuals: at $=\Phi$ txt $r t=v-a t$ $\mathrm{t}=\mathrm{t}+1$;

Step 5: Given the reconstruction error threshold $\delta$, if $\|\mathrm{rt}\| 2<\delta$,jump to output ;

Step 6: if $\mathrm{t}<\mathrm{K}$, return to step1.

Noticing that amusing we chose $x$ signal which arbitrary sparsity is $\mathrm{m}$ from $R^{d}$, and $\Phi$ is a $\mathrm{N} \times \mathrm{d}$ dimension Gaussian matrix, then we execute the TOMP $\mathrm{v}=\Phi \mathrm{x}$. If the residuals ' $\mathrm{r} \mathrm{m}$ ' is equal to zero after $\mathrm{m}$ iteration, it is considered that TOMP could complete the recovery of the original signal $\mathrm{x}$, otherwise this represents that TOMP algorithm is failure.

Compared with the OMP algorithm, TOMP has added the reconstruction error threshold $\delta$ which is determined by the demand for the actual structure of the health damage detection. For each iteration in the signal reconstruction process, it is necessary to compared the reconstructed result and the original one with $\delta$, if $\|\mathrm{rt}\| 2<\delta$, then we can stop the reconstruction and jump to output. In the other words, if it met with the demand that we can end prematurely instead of iterative $\mathrm{K}$ times. In a word, TOMP could improve the convergence speed of the algorithm effectively while maintaining the reconstruction accuracy which has improved the reconstruction efficiency.

\section{Performance Evaluation}

\subsection{Evaluation Standards for CS Application in SHM}

Compression ratio $(\mathbf{C R})$ : The compression ratio is one of the indicators to measure the degree of data compression whose definition is the compression ratio between the original signal data quantity and the compressed data amount written as the follows (6):

$$
C R=N_{o} / N_{c o}
$$

Where $N_{o}, N_{c o}$ denote the signal data quantity and compressed data amount. The larger the $\mathrm{CR}$ is, the better compression performance will be with smaller traffic load on the network.

Reconstruction error $\xi$ : Reconstruction error is on behalf of the similarity degree of the reconstructed signal and the original one. It is an import indicator to measure the effects of data decompression after refactoring which formula is (7):

$$
\xi=\frac{\|\hat{x}-x\|_{2}}{\|x\|_{2}}
$$

Where $\hat{x}, x$ separately indicated the reconstructed signal and the original one. The smaller the reconstruction error is, the higher the data recovery accuracy of the compressed sensing reconstruction algorithm is. 


\subsection{Simulation Results}

In order to get the effective and real data of the experiments, we designed a data acquisition experimental system which sensor node is a common node without compression function. The experimental system which is shown on the Figure 1 consists of a PXI data acquisition system, YE5850 charge amplifier, KH7602 broadband power amplifier and LF-21M rust-proof aluminum pasted piezoelectric patch. The data acquisition system PXI is usually for the gathering of the monitoring signal with the maximum sampling frequency $10 \mathrm{MHz}$. The sampling frequency of this experiment is $1 \mathrm{MHz}$ and collected 1024 points.

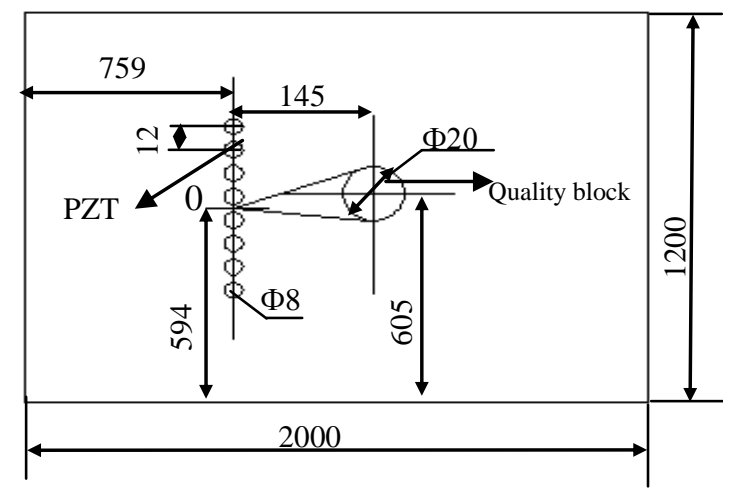

Figure 1. Schematic Diagram of the Experimental Specimen

In the data collection process, the whole size of the original data is about $208 \mathrm{M}$. And the original real data in structural health monitoring will be used to validate the compressed sensing technology. Besides, in this experimental verification process, we choose Gaussian random matrix as the measurement matrix which has the most irrelevant with any sparse base. Moreover, each element independently meets with $(0,1 / \mathrm{N})$ normal distribution. Probability density function is (8):

$$
p(x)=\frac{1}{\sqrt{2 \pi N}} e^{-x^{2} N / 2}
$$

Figure 2 describes the process of the reconstruction for the original signal without noise which has been compressed by CS. The signal length is $\mathrm{N}=1024$, and the hardware threshold method SORH=' $h$ ' is adopted. Moreover the selection of the threshold value is based on the Stein unbiased risk estimation threshold (rigrsure) which is TPTR='rigrsure'. Let the Gaussian random matrix as the measurement matrix and transform base is the Haar wavelet orthogonal transform base. Reconstruction algorithm is TOMP. 

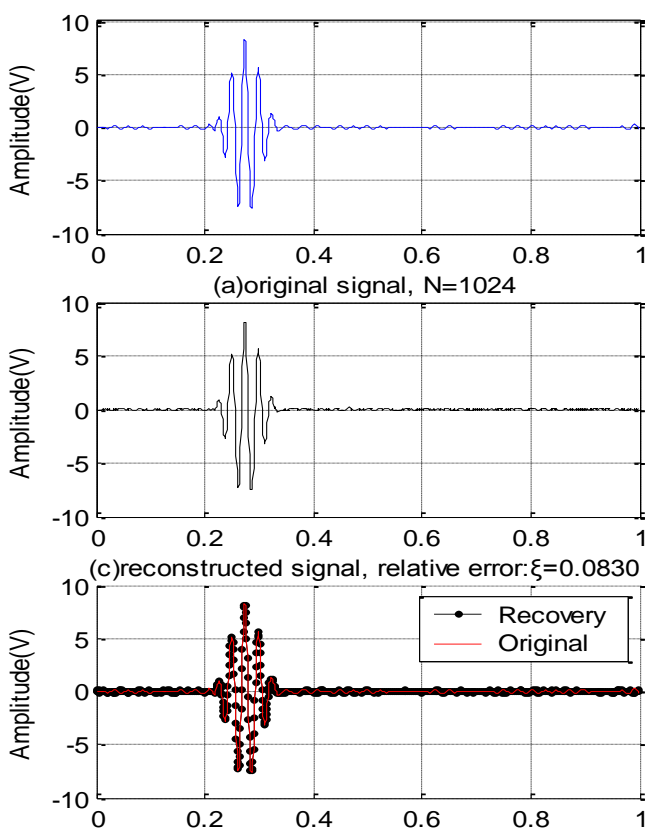

(e) the superimposed contrast between reconstructed signal and the original one
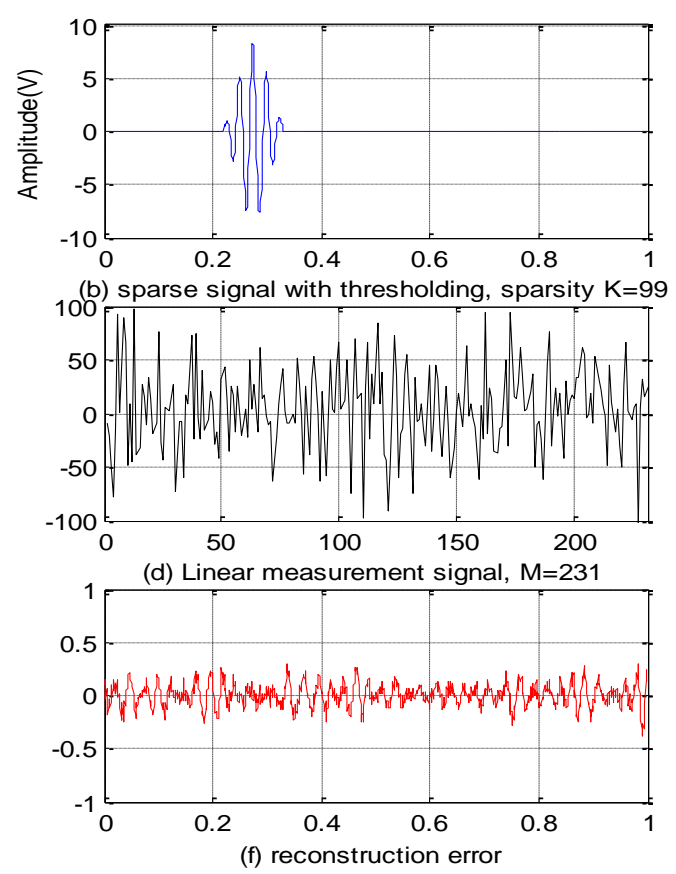

Figure 2. The Analysis Chart for the Reconstruction of the Signal without Noise

It can be seen from the Figure 2, the signal sparsity after thresholding is $\mathrm{K}=99$; the number of observations is $\mathrm{M}=231$ which dropped from the $\mathrm{N}$-dimensional to $\mathrm{M}$-dimensional; the $\mathrm{CR}$ is $1024 / 231=4.4329$; and relative error of the reconstructed signal is $\xi=0.0830$ and the absolute error of the reconstructed signal with the original signal is during [0.3262, 0.3038]. On the figure 2(f), the absolute error of about $80 \%$ sampling point distributed in \pm 0.13 . In a word, CS could achieve a higher $\mathrm{CR}$ and accuracy of the signal reconstruction for the original signal without noise from the experimental results.

\section{Conclusions and Future Works}

In this article, the potential of CS for compressing sparse data for SHM is investigated by using real sound vibration data on the aircraft. Through the all kinds of experiments and theoretical analysis, we found the specific CS technology for the SHM. From the simulation, this technology is not only able to ensure the accuracy of the data, but also improve the real-time data while reducing the load on the data transfer which provides a better way for the WSNs on the SHM applications. As a result, it has a good prospect application. Of course, this paper is not a perfect. The studies in the future are to improve the measurement matrix.

\section{Acknowledgments}

This work was supported by the Natural Science Foundation of the Jiangsu Higher Education Institutions of China (No. 11KJB520011) and a Project Funded by the Priority Academic Program Development of Jiangsu Higher Education Institutions (the PAPD). It was also supported by the Industrial Strategic Technology Development Program (10041740) funded by the Ministry of Trade, Industry and Energy (MOTIE Korea), and by the Natural Science Foundation of Jiangsu Province (No. BK2012461). Professor Jeong-Uk Kim is the corresponding author. 


\section{References}

[1] Y. Zhang and J. Li, "Wavelet-Based Vibration Sensor Data Compression Technique for Civil Infrastructure Condition Monitoring”, Journal of Computing in Civil Engineering, vol. 20, no. 6, (2006), pp. 390-399.

[2] K. K. Chintalapudi, "Design of wireless sensor network based structural health monitoring systems", Los Angeles: Univ. of Southern California, (2006).

[3] Y. F. zhang and J. Li, "Linear predietor-based lossless compression of vibration sensor data: systems appraoch", ASCE Joumal of Engineering Mechanies, vol. 133, no. 4, (2007), pp. 431-441.

[4] D. Donoho, "Compressed sensing", IEEE Trans Information Theory, vol. 52, no. 4, (2006), pp. 1289-1306.

[5] E. Candes, "Compressive sampling", Proceedings of International Congress of Mathematicians, Madrid, Spain: European Mathematical Society Publishing House, (2006), pp. 1433-1452.

[6] J. Ma and M. Y. Hussaini, "Extensions of compressed imaging: flying sensor, coded mask, and fast decoding”, IEEE. T. Instrum. Meas, vol. 60, no. 9, (2011), pp. 3128-3139.

[7] Y. Huang, J. L. Beck, S. Wu and H. Li, "Robust Diagnostics for Bayesian Compressive Sensing Technique in Structural Health Monitoring", Structural Health Monitoring (SHM), vol. 1, no. 2, (2011), pp. 103-110.

[8] D. Mascarenas, D. Hush, J. Theiler and C. Farrar, "The Application of Compressed Sensing to Detecting Damage in Structures", The 8th International Workshop on Structural Health Monitoring, Stanford University, (2011).

[9] E. Candes and T. Tao, "Near optimal signal recovery from random projections: Universal encoding strategies", IEEE Transactions on Information Theory, vol. 52, no. 12, (2006), pp. 5406-5425.

[10] E. Candes and T. Tao, "Decoding by linear programming", IEEE Transactions on Information Theory, vol. 51, no. 12, (2005), pp. 4203-4215.

[11] E. Candes, J. Romberg and T. Tao, "Robust uncertainty principles: exact signal reconstruction from highly incomplete frequency information", IEEE Transactions on Information Theory, vol. 52, no. 2, (2006), pp. 489-509.

[12] R. G. Baraniuk, "Compressive sensing”, IEEE Signal Processing Magazine, vol. 24, no. 4, (2007), pp. 118-121.

[13] S. Mallat and Z. Zhang, "Matching Pursuits with Time-frequency Dictionaries", IEEE Transactions on Signal Processing, vol. 41, no. 12, (1993), pp. 3397-3415.

[14] J. A. Tropp and A. C. Gilbert, "Signal Recovery from Partial Information by Orthogonal Matching Pursuit", www.personal.umich.edu/_jtropp/papers/TG05-Signal-Recovery.pdf, (2005).

[15] S. S. Chen, D. L. Donoho and M. A. Saunders, "Atomic decomposition by basis pursuit", SIAM Journal on Scientific Computing, vol. 43, no. 1, (2001), pp. 129-159.

[16] S. Ji, "Bayesian compressive sensing", IEEE Transactions on Signal Processing, vol. 56, no. 6, (2008), pp. 2346-2356.

\section{Authors}

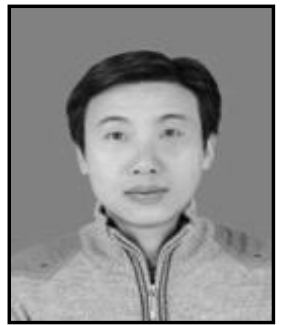

Sai Ji, is an associate professor in Computer Sciences at Nanjing University of Information Science \& Technology, China. He received his Bachelor (NUIST, China, 1999), Master (NUAA, China, 2006). His research interests are in the areas of Data Mining, Computer Measurement and Control and Wireless sensor networks. He has published more than 20 journal/conference papers. He is principle investigator of three NSF projects.

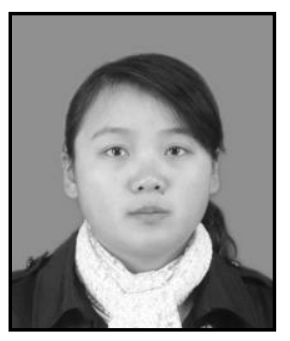

Liping Huang, is a graduate student in Nanjing University of Information Science \& Technology currently. She received her bachelor's degree in Network Engineering from Nanjing University of Information Science \& Technology in 2011. Her research interest includes network security, mobile computing, machine learning, CS technology applications and WSNs. 


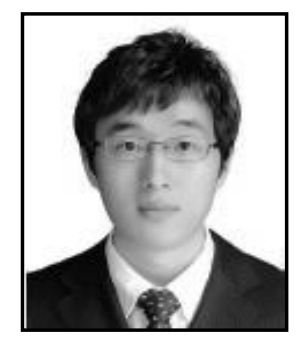

Jian Shen, received the B.E. degree from Nanjing University of Information Science and Technology, Nanjing, China, in 2007 and the M.E. and Ph.D. degrees in Computer Science from Chosun University, Gwangju, Korea, in 2009 and 2012, respectively. Since late 2012, he has been a faculty member in the School of Computer and Software at Nanjing University of Information Science and Technology, Nanjing, China. His research interests include computer networking, security systems, mobile computing and networking, ad hoc networks and systems, and ubiquitous sensor networks.

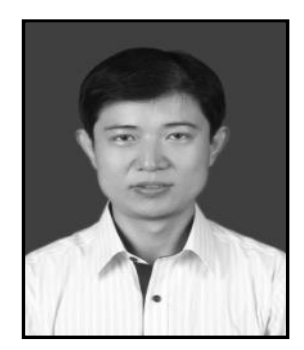

Jin Wang, he received the B.S. and M.S. degree from Nanjing University of Posts and Telecommunications, China in 2002 and 2005, respectively. He received Ph.D. degree from Kyung Hee University Korea in 2010. Now, he is a professor in the Computer and Software Institute, Nanjing University of Information Science and Technology. He has published more than 120 journal and conference papers. His research interests mainly include routing protocol and algorithm design, performance evaluation and optimization for wireless sensor networks.

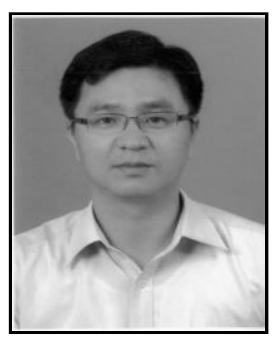

Jeong-Uk Kim, he obtained his B.S. degree from Seoul National University in 1987, M.S. and Ph.D. degrees in Electrical Engineering from Korea Advanced Institute of Science and Technology in 1989, and 1993, respectively. He is a professor in Sangmyung University in Seoul. His research interests include smart grid demand response, building automation system, and renewable energy. 